\title{
Object Detection using IoT and Machine Learning to Avoid Accident and Improve Road Safety
}

\author{
Shrinath Oza \\ Department of Computer Engineering, \\ Dr. D.Y Patil School of Engineering Lohegaon, \\ Pune, Savitribai Phule, Pune University \\ Pune, India
}

\author{
Dr. Sunil Rathod \\ Department of Computer Engineering, \\ Dr. D.Y Patil School of Engineering Lohegaon, \\ Pune, Savitribai Phule, Pune University \\ Pune, India
}

\begin{abstract}
At present situation the human beings are going through many accidents during the road way transportation. Simultaneously they lose their life and significant properties in those accidents. Most of the Indian roads in rural and suburban's are not ideal for driving due to faded lanes, irregular potholes, inappropriate and unseen road signs, which caused many accidents, lost lives and caused serious damage to vehicles. The most difficult task is to detect obstacles on the highway. The basic concept is to design a system that has the effect of detecting the presence of an obstacle in the track of the vehicles. In the proposed work, the Raspberry Pi Camera module is used for object detection and image acquisition. A thorough study is performed on a test image to test the best algorithm suitable for detecting image boundaries. The framework performs preprocessing utilizing the Mean Subtracted Difference Enhancement (MSDE) strategy and afterward segmentation is performed. The classification is done by using proposed Advanced Classifier for the detection of objects. The system can classify objects like vehicles, animals, humans, etc. Once the object is detected the system informs the user to slow down the vehicle through a voice message. A sufficient analysis is carried out to consolidate the results obtained. The result analysis shows that proposed system is more precise and consumes less time than existing system.
\end{abstract}

\section{Keywords- Raspberry Pi, Object detection, Image processing, IoT, Segmentation}

\section{INTRODUCTION}

One major issue that all the developed countries are facing today is death and injuries because of road accidents. In India, most people are killed in road accidents rather than diseases. According to the study, about $45 \%$ of persons died in road accidents within a year. Highway obstacle detection is one of the most complex and complex tasks in real-time systems of the machine. In 1988 of Delco systems operations, Goleta, California, was the first who developed the first obstacle detection system, which detects and warns the driver about obstacles on the road. After several years at Santa Barbara research and Corp. (SBRC), California initially establishes obstacle detection methods, which use ultrasound, infrared lasers, and radar of the implant. In recent decades, much Driving Assistance Systems was developed to enhance the safety of the driver and passengers. Introduced a lot of obstacle detection systems that will take the various active sensors such as millimeter-wave radar, LIDAR sensor, infrared laser sensor, and ultrasonic sensor Embedded technology has gained immense momentum in the automotive industry. To ensure safety and transport efficiency, intelligent motor cars used today. The obstacle detection system is intended to diminish the damaging effects of an accident. This can be done effectively using new technologies such as radar, lasers, cameras and ultrasonic sensors to detect obstacles on the front or back of the vehicle. The United Nations General Assembly has approved a 10 year period from 2010 to 2020 as the decade of action to be taken to assess road safety [1].

Previously, various obstacle detection systems in automotive systems widely used infrared sensors as a proximity sensor to prevent obstacles. Since IR sensors have non-linear behavior and their basic concept depends on reflection from the surrounding object, some error always creeps in, making them unsuitable for applications that require high accuracy. Therefore, these sensors are only recommended for measuring short distances up to 25 $\mathrm{cm}[2]$.

The development of an automated driver guidance system is extremely significant in the context of Indian road conditions. It is difficult for the driver to drive the vehicle due to sudden potholes or bumps or sharp turns when road signs are not very visible or absent most of the time. Let's assume that if there is a system with an integrated motion camera and a built-in on-Board computer with the vehicle, a simple driver guidance system can be developed based on frame analysis of motion frames, and thereby generating alarms accordingly. So driving can be done quite easily. Road image analysis is an extremely significant feature of an automated driver support system. High-quality real-time analysis of road data is the cornerstone of any modern transport system. Up until now, a significant part of the analysis is done manually, and the utilization of image processing strategies for qualitative analysis is still at a beginning period [3].

In this paper, Accident Avoidance and Improving Road Safety with Use of Raspberry Pi for Object Detection system are proposed. We propose a method for detecting obstacles using a monocular camera mounted on a vehicle that takes light variations of the scene on the road ahead and analyzes the resulting images to perform obstacle 
detection. The camera is used to capture continuous realworld images. According to the images available through the camera, these images can be transmitted to the raspberry pi to perform the control actions of the car. The framework performs preprocessing utilizing the Mean Subtracted Difference Enhancement (MSDE) strategy and afterward segmentation is performed. The classification is done by using proposed advanced classifier. The system can classify objects like vehicles, animals, humans, etc. Once the object is detected the system informs to the user to slow down the vehicle through a voice message. After receiving information about the obstacle, drivers can quickly and accurately respond to take appropriate action to prevent road accidents.

\section{REVIEW OF LITERATURE}

The various researchers conducted studies on object detection and tracking systems. Z. Zhang, Zhihai He, G. Cao, and W. Cao [1] propose an accurate method for animal object detection. To develop a new approach to the formation of proposals for areas of animal origin using a multi-level graph section in the space-time domain. Then the author develops a method for verifying cross-frame time patches to determine whether these regional proposals are true animals or background patches. They construct an effective feature description for animal detection using collaborative deep learning and a histogram of oriented gradient features encoded by Fisher vectors. R. V.Nahari, R.Alfita, H.Anam, K. A.Wibisono and M. Pramudia [2],develop a system for detecting road damage by processing images and mapping road damage, which is stored in a database. This system uses a webcam to capture the road in real-time, the webcam is located in front of the car, and the GPS module is used to mark the location of the road damage, which is controlled by the Arduino Uno microcontroller.

R. Pavithra and M. Jagadeesan [3], paper present a simple and inexpensive system for automatic detection of animals on highways to prevent collisions of vehicles with animals and objects using image processing and computer vision. Also presented is a method for determining the distance to the animal in real units of measurement using a camera mounted on a vehicle. The presented system is trained on more than 2200 images consisting of positive and negative images, as well as tested on different video clips of animals on the roads with dissimilar vehicle speeds.

M. Tiwari and Dr. R.Singhai [4], Object detection and tracking is one of the most important areas of research related to routine changes in object movement and scene size, occlusions, changes in appearance, and changes in ego movement and lighting. In particular, object selection is a vital role in tracking objects. This is due to many real-time applications, such as car perception, video surveillance, and so on. To overcome the problem of detection, tracking is related to the movement of the object and its appearance. Most algorithms focus on the tracking algorithm to smooth out the video sequence. On the other hand, only a few methods use previously available information about the shape of the object, color, texture, and so on. In this paper, we discuss and analyze the tracking algorithm that combines the above parameters of objects. The purpose of this work is to analyze and review the previous approach to tracking and detecting objects using video sequences at various stages. Also, identify the gap and suggest a new approach to improve object tracking on the video frame.

R. Kasde and G. Gugapriya [5], the article is to improvements of cars to make them more interactive and intelligent to prevent accidents on the roads. As a safety improvement, the multi-sensor Control area network (CAN) system is coupled to the Engine control unit (ECU) using the ARM-7 microcontroller. To prevent accidents, various sensors are used to monitor the driver's fatigue level, heart rate, alcohol level, obstacle detection, and sudden collisions. Global positioning system, GSM and CAN technology for faster communication make the system completely reliable, safe and stable, and it achieves the expected result of real-time data analysis very effectively to provide a more secure drive.

V. Kumaravel, and R. Sharmila gowri [6], designed a system with the help of two main processors (Raspberry pi and PIC microcontroller). Digital processing plays a vital job in the sign capturing and detection system. Image processing algorithms take the essential action, resizing the captured signs. Raspberry pi Camera port is used to capture traffic signs image enhancement techniques. The embedded system is a small computer platform to study the characteristics of speed signs. In daylight, vision time takes shape analysis for recognizing signs using edge detection algorithms. After a night trip detects speed signs using color enhancement techniques.

P.Ulhe, S.Gawande, and K.Taksande [7], the author study the Indian roads, to suggest the method to detect lanes, potholes, and road signs and their classification and to suggest automated driver guidance mechanism. Using processing based on Edge detection and Hough Transform has been developed to help the driver of lane-departure decision-making process, reduce the loss of concentration and avoid accidents while driving.

M. Bendjaballah, S. Graovac and M. A. Boulahlib [8], the author implements system based on processing too many applications in the domain of motion control of robots and autonomous vehicles. The current paper focused on the solution of the problem, which precedes the implementation of automated object detection system to avoid on-road obstacles - how to detect them, to track the sequence of images and recognize which of them are stationary, incoming, or outgoing from the camera.

Fares Jalled [9], this article is about developing OpenCV-Python code using the Haar Cascade algorithm for object and Face Detection. Currently, Unmanned Ariel vehicle (UAVs) is used to detect and attack infiltrated ground targets. The main disadvantage of this type of UAV is that the object hits the UAV, because sometimes the object is not detected properly. It aimed to avoid such undesirable collisions and damage to the UAV. The UAV is also used for surveillance, which uses the Viola jones algorithm to detect and track humans. The adoption of this algorithm revealed that the Cascade object detector will function train to train the algorithm function. The main advantage of this code is the reduction of processing time. The Python code was tested with the help of an available 
database of videos and images, and the output was validated.

\section{PROPOSED SYSTEM}

\section{A. Proposed System Architecture}

Detecting road obstacles is one of the most challenging real-time tasks for Autonomous vehicle navigation systems. The main idea is to develop an effective system for a real-time environment that detects the presence of obstacles on the vehicle track. Highway accidents are a human tragedy. They call high human suffering and momentary value, in terms of sad deaths, injuries, and loss of possible income. The calendar year 2017 was close to 5 lakh highway accidents in India, which leads to more than 1.3 lakh deaths and cause injuries 5.2 lakh people. These numbers translated into one road accident in every minute and one highway accident death every four minutes. Now it is clear that the main causes of highway accidents, the driver related. So we can develop object detection systems that help the driver, it can reduce the number of accidents, absence, and here is a study which is directed at Highway Safety, and safety. When an object is identified, drivers are notified via voice message.

Object detection and tracking are key features in many computer vision applications, with uses in autonomous cars, medical diagnostics, surveillance, and industrial automation, among others. This is an area of research that has made significant progress in recent years. With the increase in computing power of modern computers and equipment and the increase in data availability, complex models can achieve high levels of accuracy and processing time. With the right model and powerful hardware, realtime performance can be achieved. Figure 1 shows the Accident avoidance and improving road safety with the use of Raspberry Pi for the object detection system.

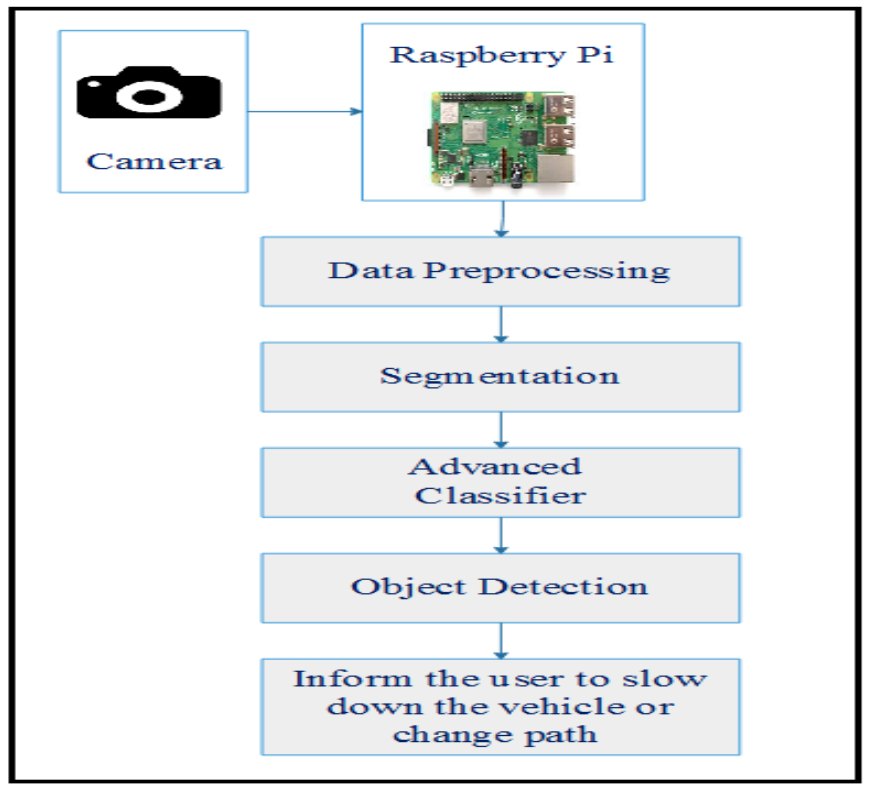

Fig. 1. Proposed System Architecture

The Raspberry $\mathrm{Pi}$ is a small single-Board computer, similar to a credit card, with a base size of $85 \mathrm{~mm}$ x 56 mm, developed by the Raspberry Pi Foundation.
The Raspberry Pi can quickly capture a sequence of images using its capture port with a JPEG encoder. Using the capture sequence method our Raspberry Picamera can capture images in the rate of $20 \mathrm{fps}$ at a $640 \times 480$.

The camera is used to capture continuous real-world images. According to the images available through the camera, these images can be transmitted to the raspberry pi to perform the control actions of the car.

The system performs data preprocessing using the Mean Subtracted Difference Enhancement (MSDE) method. Data processing is a way to convert an image into digital form and perform some operations on it to get an improved image or extract some useful information from it. This is a type of signal distribution in which the input is an image, such as a video frame or photo, and the output can be an image or characteristics associated with this image. Typically, an image processing system involves processing images as two-dimensional signals by applying already set signal processing techniques to them.

Image segmentation is the process of dividing a digital image into several segments (a set of Pixels, also called image objects). The purpose of segmentation is to simplify or change the representation of an image to something more meaningful and easier to analyze.

The classification is done by using advanced object detection classifier. The system can classify objects like animals, humans, vehicles, etc. Once the object is detected the system inform to the user to slow down the vehicle through a voice message.

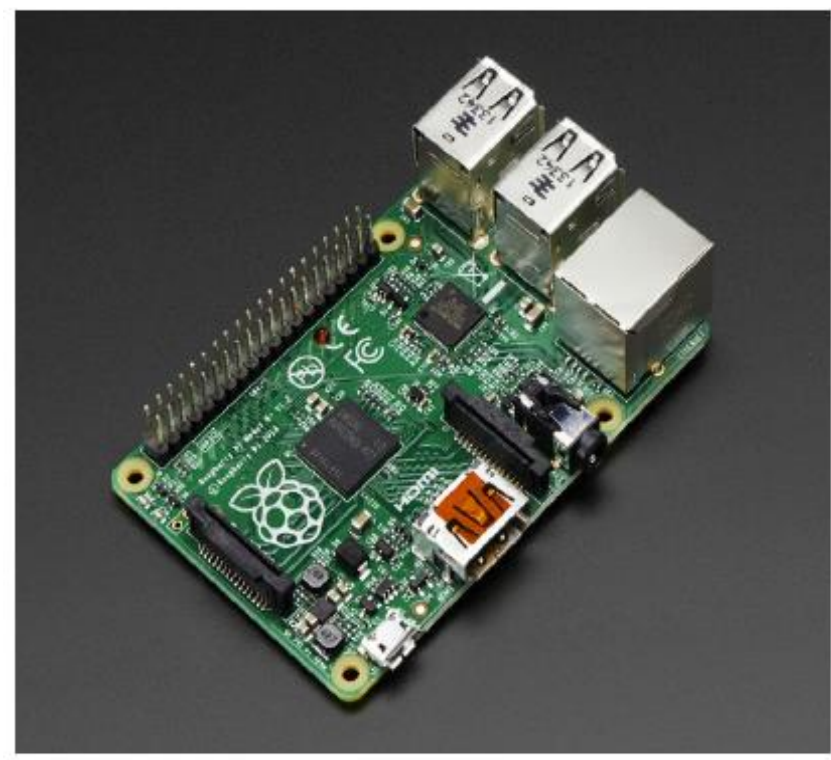

Fig. 2. Raspberry Pi Model

\section{B. Algorithm}

1) Algorithm 1:Deep Convolutional Neural Network (DCNN) Algorithm

1. Input it $(32 \times 32 \times 3)$ will hold the raw pixel values of the image. 
2. The CONV layer computes the dot product between each weight and the small area they are connected to the input volume, which is connected to the local area of the input, which could be a volume such as $(32 \times 32 \times 12)$ if you decide to use 12 filters.

3. The RELU layer will apply the activation element-wise function, such as the maximum threshold $(0, x)$ at zero. The volume size remains unchanged $(32 \times 32 \times 12)$.

4. A down sampling performed by the pool layers operation along the spatial dimension (width,

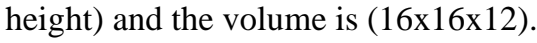

5. Fully connected (FC) layer, it computes the class scores, resulting in a volume of size (1x1x10).

\section{2) Algorithm 2: YOLO (You Only Look Once)}

The YOLO framework deals with object detection in a different way. It takes the entire image in a single instance and predicts the bounding box coordinates and class probabilities for these boxes. YOLO also understands generalized object representation. The limitation of YOLO algorithm is that it struggles with small objects within the image, for example it might have difficulties in detecting a flock of birds. This is due to the spatial constraints of the algorithm.

The Framework functions of YOLO are

1. First takes an input image

2. The framework then divides the input image into grids (say a 3 X 3 grid)

3. Image classification and localization are applied on each grid.

4. YOLO then predicts the bounding boxes and their corresponding class probabilities for objects (if any are found, of course).

\section{3) Algorithm 3:Advanced Classifier (Proposed Algorithm)}

1. Take an input image.

2. Convolution batch normalization and Rely activation is done on image.

3. Pooling is performed on image, pooled image is fully connected.

4. Extended image is obtained.

5. Recurrent function is performed on extended image.

6. Finding object detection is done.

\section{Mathematical Model}

Let ' $S$ ' be the system that detects and classifies the object in front of vehicle using machine learning.

$$
\begin{aligned}
& \mathrm{S}=\{\mathrm{I}, \mathrm{O}, \mathrm{F}\} \\
& \mathrm{I}=\{\text { Input to train the system }\} ; \\
& \mathrm{O}=\{\text { Output of the system }\} ; \\
& \mathrm{F}=\{\text { Functions of the system }\} ;
\end{aligned}
$$

1) $I=\{I 1\}$;

$$
\begin{aligned}
& \mathrm{I} 1=\{\text { Image as input to the Raspberry } \mathrm{P} i\} \\
& \mathrm{F}=\{\text { Camera capture the image and send to } \\
& \text { Raspberry } \mathrm{Pi}\}
\end{aligned}
$$

2) Advanced Classifiers:

Apply Advanced Classifier ()$=\{$ Performs Advanced Classifier\};

$$
\begin{aligned}
& i_{t}=\sigma\left(w_{i}\left[h_{t-1}, x_{t}\right]+b_{i}\right)(1) \\
& f_{t}=\sigma\left(w_{f}\left[h_{t-1}, x_{t}\right]+b_{f}\right) \\
& o_{t}=\sigma\left(w_{o}\left[h_{t-1}, x_{t}\right]+b_{o}\right)
\end{aligned}
$$

Where,

$i_{t}=$ input gate, $f_{t}=$ Forget gate, $o_{t}=$ Output gate.

$\sigma=$ Sigmoid function, $w_{x}=$ weight for the respective gate (x) neurons, $h_{t-1}=$ output of the previous block (at timestamp-1), $x_{t}=$ input at current timestamp, $b_{x}$ $=$ biases for the respective gates $(\mathrm{x})$.

Advanced Classifier $=\{$ Classifies the image and detect the object\};

Detection ()$=$ Detect the object and inform the user to slow down the vehicle or change the path\};

3) $\mathrm{O}=\{\mathrm{O} 1\}$;

$\mathrm{O} 1=\{$ Detection: Detect objects $\}$;

\section{RESULT AND DISCUSSION}

\section{A. Experimental Setup}

The 64-bit Operating System, x64-based processor and Python technology is used to develop the proposed system. The system utilizes the PyCharm as a development tool. The system requires Intel(R)Core(TM)i5-6200U CPU @ $2.30 \mathrm{GHz}$ and camera. Any standard machine can run the application.

\section{B. Result}

Today, images and videos are everywhere. There are billions of online photo-sharing sites and social networks. The field of vision research is dominated by machine learning and statistics. Using images and videos to detect, classify, and track objects or events to understand a real scene. Computer programming and the development of algorithms for understanding what is in these images is the domain of computer vision. Computer vision supports applications such as image search, robot navigation, medical image analysis, photo management, and more. From a computer point of view, an image is a scene consisting of objects of interest and background 
represented by everything else in the image. The relationships and interactions between these objects are key factors in understanding the scene. The object detection field is usually performed by searching for each part of the image to locate parts whose photometric or geometric properties correspond to the target object in the training database. The main goal of this project is to develop object detection for accident avoidance and improving road safety with the use of Raspberry Pi. The system is used to detect objects in front of the car using the camera module to prevent accidents and improve road safety using the Raspberry Pi. The Raspberry Pi is a series of small, lowcost and low-power computers developed by the Raspberry Foundation, a charity based in the UK.

The system uses Camera-trap and popular CDnet dataset for evaluation purposes. The system focuses on accurate and reliable object detection and segmentation from camera-trap images. We establish a camera trap kit for assessing object from highly overcrowded natural scenes. This dataset contain 800 camera trap images. Table I shows the training time comparison between proposed Advanced Classifier and the existing CNN and YOLO algorithm. Figure 3 shows that the proposed Advanced Classifier requires less training time than the existing algorithms. Advanced classifier requires 80 minutes for training.

TABLE I.TRAINING TIME COMPARISON

\begin{tabular}{|c|c|}
\hline Algorithm & Time in Minute \\
\hline Advanced Classifier & 80 \\
\hline YOLO & 90 \\
\hline CNN & 120 \\
\hline
\end{tabular}

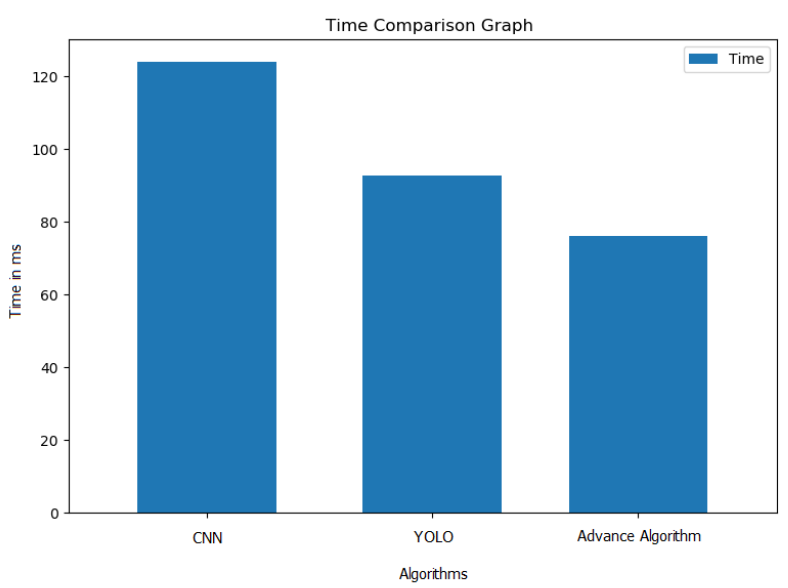

Fig. 3. Training Time Comparison Graph

Table II shows the accuracy comparison between Advanced Classifier and the existing CNN and YOLO algorithm. Figure 4 shows that the proposed advanced classifier is more accurate than the existing $\mathrm{CNN}$ and
YOLO. Advance Classifier shows a higher $80 \%$ of accuracy.

\section{TABLEII.ACCURACY COMPARISON}

\begin{tabular}{|c|c|}
\hline Algorithm & Accuracy in \% \\
\hline Advance Classifier & 80 \\
\hline YOLO & 68 \\
\hline CNN & 60 \\
\hline
\end{tabular}

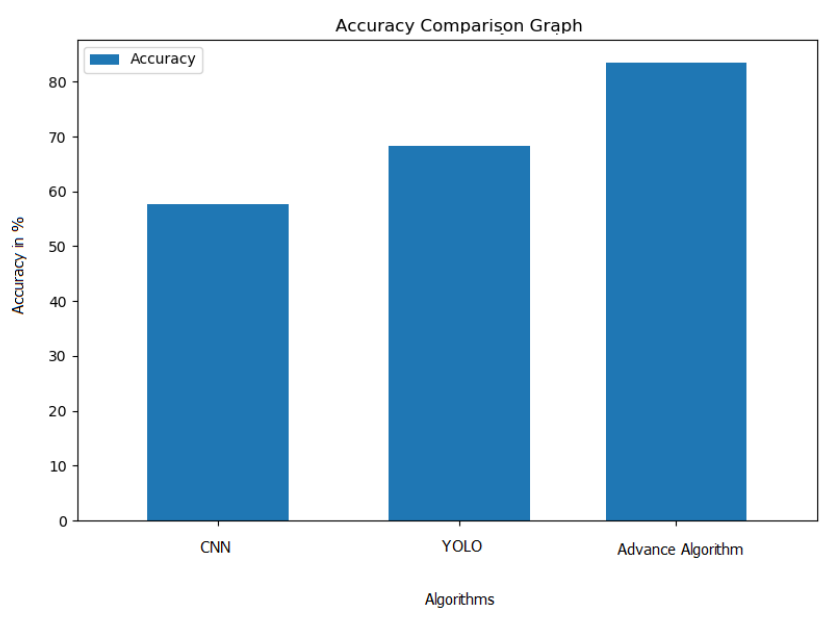

Fig. 4. Accuracy Graph

\section{CONCLUSION}

Driving with too short of a safety distance between the vehicles is a common problem in road traffic, which often results in traffic accidents as a consequence. The number of vehicles is increasing day by day and because different road conditions, accidents can occur between vehicles, human as well as animals, so object detection is very important. The main goal of this project is to develop object detection for accident avoidance and improving the Road Safety with Use of Raspberry Pi. A user-friendly visualization approach of the detected images is provided in this paper.

The main purpose of the system is to implement the real-time objects detection system on a Raspberry Pi to avoid accidents and improving road safety. In which the system performs preprocessing using the mean subtracted difference extension (MSDE) and then segmentation is performed. Classification is done using proposed advanced classifier. The system can classify objects such as animals, people, cars, etc. After the object is detected the system will notify the user to slow down the car via voice message. The result analysis shows that proposed system is more precise and consumes less time than existing CNN and YOLO object detection methods. 


\section{REFERENCES}

[1] Zhi Zhang, Zhihai He, Guitao Cao, and Wenming Cao, "Animal Detection From Highly Cluttered Natural Scenes Using Spatiotemporal Object Region Proposal sand Patch Verification", IEEE 2016

[2] Rosida Vivin Nahari, Riza Alfita, Hairul Anam, Kunto AjiWibisono, Mirza Pramudia,"Camera-Based Road Damage Detection System with Edge Detection Algorithm", ICCSET 2018

[3] R. Pavithra, M. Jagadeesan, "Automatic Object Detection and Collision Avoidance System using Cascaded Random Classifier", IJRASET 2018

[4] Mukesh Tiwari, Dr. RakeshSing3hai, "A Review of Detection and Tracking of Object from Image and Video Sequences", International Journal of Computational Intelligence Research, 2017

[5] Ratnaprabha Kasde and G. Gugapriya, "Accident Avoidance System using CAN", Indian Journal of Science and Technology, August 2016
[6] V.Kumaravel, R.Sharmila gowri, "Raspberry Pi Based Road Sign Recognition System with Additional Safety Parameters Using Image Processing", International Journal of Engineering and Management Research, 2017

[7] PRANJALI ULHE, SAMTA GAWANDE, KAJOL TAKSANDE, "IMAGE PROCESSING ON ROAD DETECTION", International Journal of Advances in Science Engineering and Technology 2015

[8] Mourad Bendjaballah, Stevica Graovac and Mohammed Amine Boulahlib, "A classification of on-road obstacles according to their relative velocities", Bendja ballahet al. EURASIP Journal on Image and Video Processing (2016)

[9] Fares Jalled,"Object Detection Using Image Processing”, 1611.07791v1 [cs.CV]23 Nov 2016

[10] Zhi Zhang, Zhihai He, Guitao Cao, and Wenming Cao, "Animal Detection From Highly Cluttered Natural Scenes Using Spatiotemporal Object Region Proposals and Patch Verification", IEEE 2016 